\title{
LETTER
}

\section{Cytokine adsorption in severe, refractory septic shock}

\author{
Pedro David Wendel Garcia ${ }^{1} \mathbb{B}$, Matthias Peter Hilty ${ }^{1} \mathbb{B}$, Ulrike Held² ${ }^{\circledR}$, Eva-Maria Kleinert ${ }^{1}$ \\ and Marco Maggiorini ${ }^{*^{*}}$ (B)
}

(c) 2021 Springer-Verlag GmbH Germany, part of Springer Nature

Dear Editor,

Septic shock and the underlying dysregulated inflammatory host-response remain major contributors to mortality in critically ill patients. In contrast to classic hemofiltration strategies, cytokine adsorption through bulk removal of cytokines has been postulated to reestablish inflammatory homeostasis, representing an attractive approach to the treatment of septic shock [1]. Nonetheless, most evidence to date is of descriptive or ambivalent character and cytokine adsorption in severe, refractory septic shock is largely unexplored [2].

To investigate the effect of cytokine adsorption on circulating interleukin (IL)-6, vasopressor requirements and intensive care mortality, patients presenting with severe, refractory septic shock, IL-6 $\geq 1000 \mathrm{ng} / \mathrm{l}$ and a vasopressor dependency index $\geq 3$, despite adequate volume resuscitation, were prospectively recruited. Cytokine adsorption was provided for three consecutive 24-h sessions initiated within $24 \mathrm{~h}$ from shock onset. Forty-eight included patients were matched to 160 patients having fulfilled the same severe, refractory septic shock criteria (e-Fig. 1). Six matching algorithms were evaluated against each other to achieve optimal balancing of covariates, leading to the choice of Genetic Matching as superior algorithm. For further specifications on the employed methodology, see e-Appendix 1.

The baseline characteristics among the 96 matched patients (48 treated with cytokine adsorption, 48 treated without) were equivalent (e-Table 1, e-Fig. 2). Patients were characterized by a SOFA score of $14 \pm 3$, profound

*Correspondence: klinmax@usz.uzh.ch

${ }^{1}$ Institute of Intensive Care Medicine, University Hospital of Zurich, Rämistrasse 100, 8091 Zurich, Switzerland

Full author information is available at the end of the article lactatemia $(5.8 \pm 4.8 \mathrm{mmol} / \mathrm{l})$ and required $0.7 \pm 0.5 \mu \mathrm{g} /$ $\mathrm{kg} / \mathrm{min}$ norepinephrine. Within the 72-h intervention period, circulating IL-6 levels $(p=0.254)$ and vasopressor requirements $(p=0.555)$ decreased irrespective of cytokine adsorption use (Fig. 1a, b, e-Table 2, e-Fig. 3). Intensive care mortality was more pronounced in patients treated with cytokine adsorption than in the control group (control: 20 (42\%), cytokine adsorption: 32 (67\%), $p=0.024$ ) as evidenced by a competing risks hazard ratio for mortality of 1.82 (95\% confidence interval, $1.03-3.2 ; p=0.038$ ) (Fig. 1c). Additional analyses are presented in e-Appendix 2.

For almost four decades, the hypothesis that quantitative removal of inflammatory mediators improves survival in sepsis has resulted in negative trials [2]. Unexpectedly however, our data reflect the results of the first multicenter trial having assessed cytokine adsorption in sepsis [3] and, despite the pathophysiological differences, of a recent trial assessing cytokine adsorption in severe patients affected by coronavirus disease 2019 (COVID19) on extracorporeal membrane oxygenation [4]. It evidenced not only no effect of cytokine adsorption on circulating IL-6 levels, but also strikingly suggested an increased mortality in the cytokine adsorption group.

Cytokines play a pivotal role in the progression of host response in sepsis. Pro-inflammatory cytokines may be associated with a deranged host response and poor outcomes during early sepsis. However, nothing persists for millions of years if it does not offer a substantial evolutionary benefit. Indeed, the dynamic interplay between pro- and anti-inflammatory cytokines is imperative to achieve tissue repair, endothelial integrity and resolution of inflammation. Indiscriminate removal of cytokines could thus lead to a perpetuation of inflammation and prothrombogenicity, leading to sustained

\section{Springer}




\section{追 Control 追 Cytokine Adsorption}

a

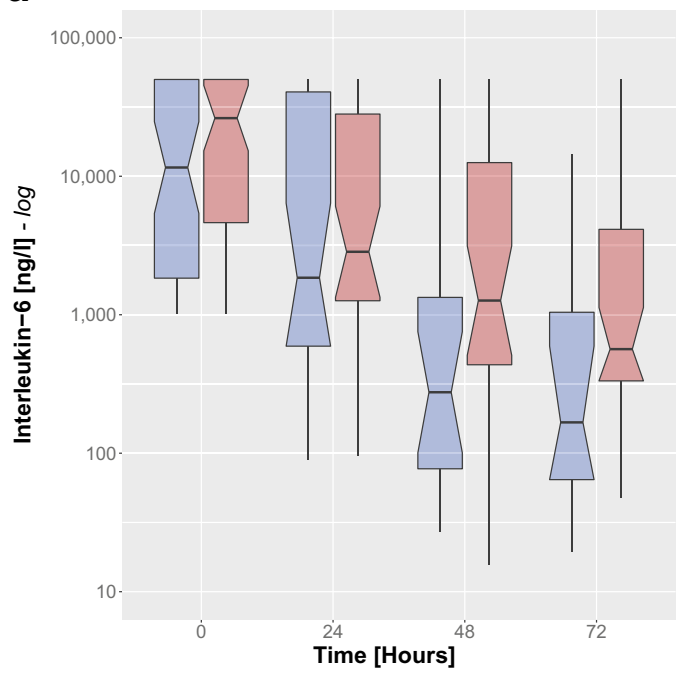

b

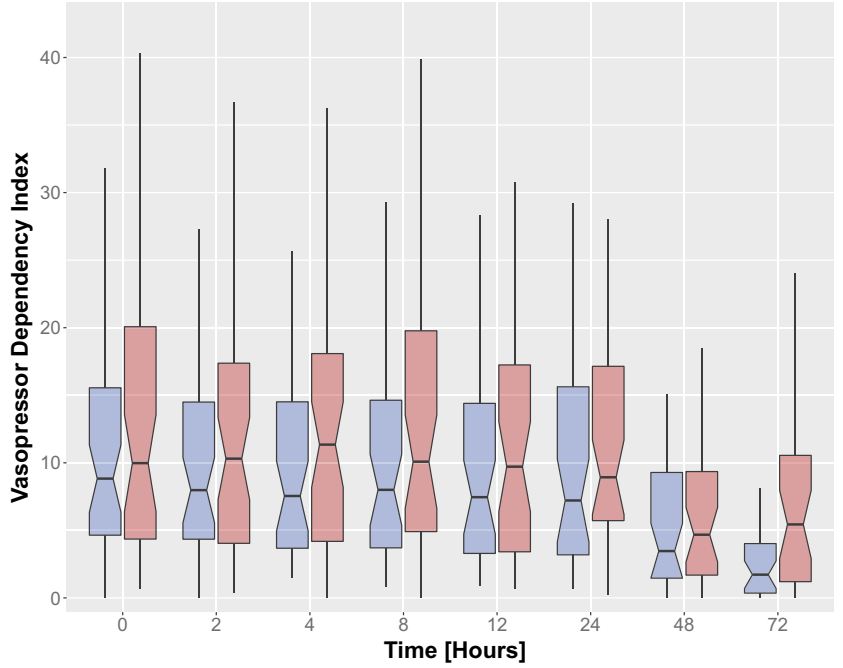

C

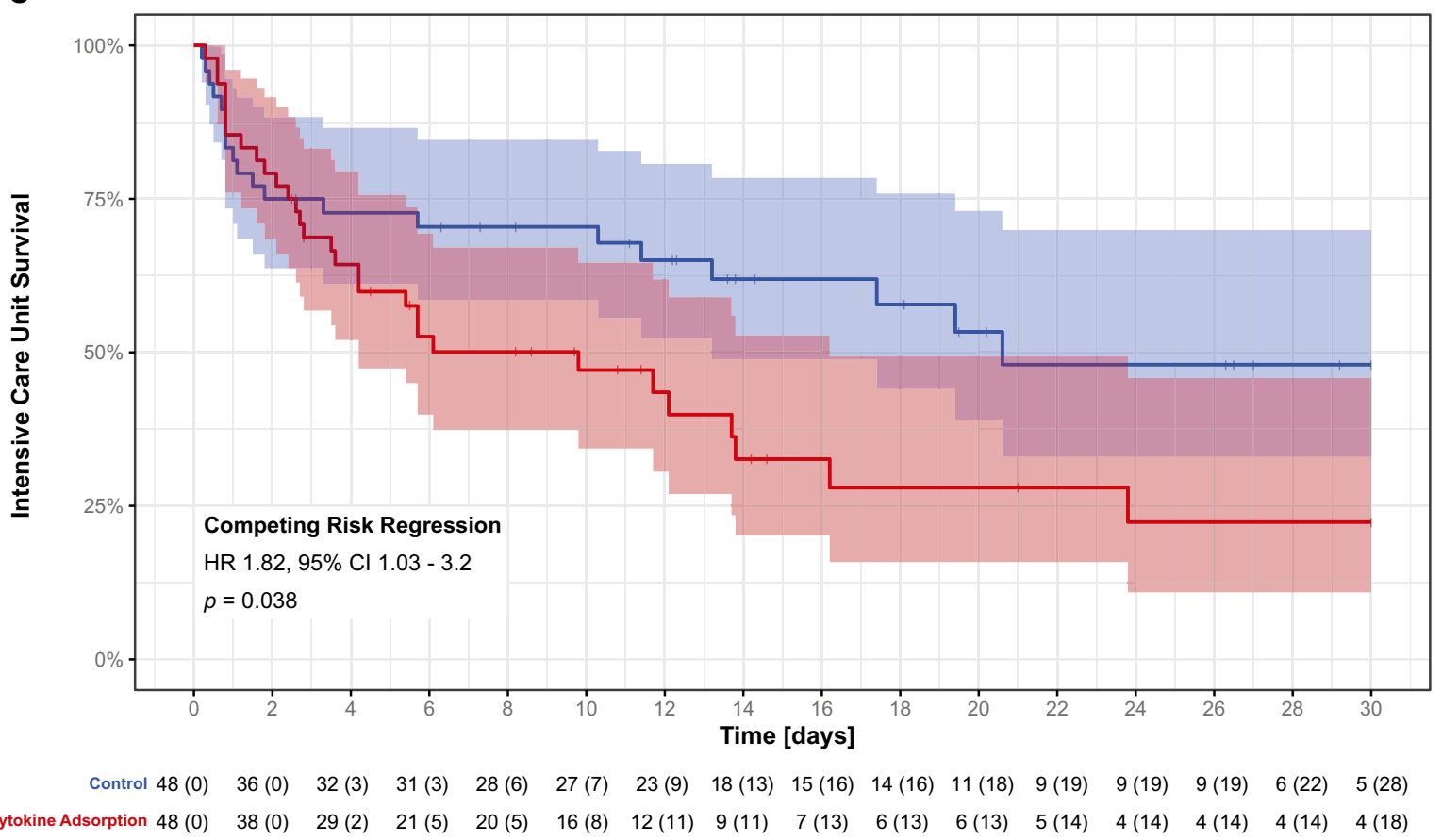

Fig. 1 Primary end points. Temporal development of (a) interleukin-6 levels and (b) the vasopressor dependency index. Depicted are notched box plots with median and interquartile range, and whiskers extending from the difference of the first quartile and 1.5 times the interquartile range, to the sum of the third quartile and 1.5 times the interquartile range. Notches represent the $95 \%$ confidence interval of the median. The $x$-axis portrays the time in hours ensuing severe, refractory septic shock onset. (c) Kaplan-Meier curves for 30-day intensive care unit survival stratified by control and cytokine adsorption group, plotted in blue and red colors, respectively. Shaded areas represent the $95 \%$ confidence interval. The $x$-axis portrays the time in days ensuing severe, refractory septic shock onset. The computed hazard ratio assesses the cytokine adsorption group using the control group as reference. The 95\% confidence interval is given in parentheses. Hazard ratios were modeled by means of a Fine and Gray competing risk analysis. Censoring reflects patients having left the intensive care unit alive. The underlying table presents the patients at risk per time point with the number of censored patients given in parentheses 
microcirculatory and mitochondrial dysfunction, ultimately promoting end-organ damage and death [5].

In conclusion, cytokine adsorption in severe, refractory septic shock was neither associated with reduced IL-6 levels nor vasopressor requirements, and lead to an increased hazard of death. The present results in conjunction with recent evidence plead against the widespread and indiscriminate use of cytokine adsorption outside of investigational settings and urge for a return to qualitative and mechanistic blood purification research in septic shock.

\section{Supplementary Information}

The online version contains supplementary material available at https://doi. org/10.1007/s00134-021-06512-0.

\section{Author details \\ ${ }^{1}$ Institute of Intensive Care Medicine, University Hospital of Zurich, Rämis- trasse 100, 8091 Zurich, Switzerland. ${ }^{2}$ Department of Biostatistics and Epi- demiology, Biostatistics and Prevention Institute, University of Zürich, Zurich, Switzerland.}

\section{Author contributions}

PDWG and MM conceived and designed the research project. PDWG and EMK handled data acquisition. PDWG, EMK and MPH accessed and verified the data. PDWG, MPH, UH and MM performed analysis and interpretation of the data. PDWG performed statistical analysis and wrote the first draft of the manuscript. MM handled funding and supervision of the research project. Al authors read, critically revised and approved the final manuscript. All authors had full access to the full data in the study and accept responsibility for the decision to submit for publication.

\section{Funding}

CytoSorbents Europe GmbH (Berlin, Germany) partially funded this study by means of an unrestricted grant. The funder had no role in the design and conduct of the study; collection, management, analysis, and interpretation of the data; preparation, review, or approval of the manuscript; and decision to submit the manuscript for publication.

\section{Availability of data and material}

All data analyzed and discussed in the framework of this study are included in this published article and its online supplementary information. The corresponding author may provide specified analyses or fully de-identified parts of the dataset upon reasonable request.

\section{Code availability}

Not applicable.

\section{Declarations}

\section{Conflicts of interest}

MM reported receiving research grants from CytoSorbents Europe $\mathrm{GmbH}$ (Berlin, Germany) and Baxter International Inc. (Deerfield, USA), as well as personal fees for his work as external consultant from Baxter International
Inc. (Deerfield, USA) and Toray Industries Inc. (Tokyo, Japan). All other authors declare no conflicts of interest.

\section{Ethical approval}

All procedures performed in studies involving human participants were in accordance with the ethical standards of the institutional and Swiss national research committee, with the 1964 Helsinki Declaration and its later amendments and with the guidelines on Good Clinical Practice issued by the European Medicines Agency. The study was approved by the cantonal ethics committee of Zurich (BASEC: ZH 201800559).

\section{Consent to participate}

Written informed consent for participation from the patient or in case of death or disability, from the next of kin or legal representative, was sought for every patient prospectively treated with the cytokine adsorber. In the historical cohort, a retrospective informed consent collection process was followed.

\section{Consent for publication}

Written informed consent for publication from the patient or, in case of death or disability, from the next of kin or legal representative was sought for every patient prospectively treated with the cytokine adsorber. In the historical cohort, a retrospective informed consent collection process was followed.

\section{Publisher's Note}

Springer Nature remains neutral with regard to jurisdictional claims in published maps and institutional affiliations.

Received: 11 June 2021 Accepted: 17 August 2021

Published online: 1 September 2021

\section{References}

1. Honore PM, Hoste E, Molnár Z, Jacobs R, Joannes-Boyau O, Malbrain MLNG, Forni LG (2019) Cytokine removal in human septic shock: where are we and where are we going? Ann Intensive Care 9:56

2. Poli EC, Rimmelé T, Schneider AG (2019) Hemoadsorption with CytoSorb ${ }^{\circledR}$. Intensive Care Med 45:236-239

3. Schädler D, Pausch C, Heise D, Meier-Hellmann A, Brederlau J, Weiler N, Marx G, Putensen C, Spies C, Jörres A, Quintel M, Engel C, Kellum JA, Kuhlmann MK (2017) The effect of a novel extracorporeal cytokine hemoadsorption device on IL-6 elimination in septic patients: a randomized controlled trial. PLOS ONE 12:e0187015

4. Supady A, Weber E, Rieder M, Lother A, Niklaus T, Zahn T, Frech F, Müller S, Kuhl M, Benk C, Maier S, Trummer G, Flügler A, Krüger K, Sekandarzad A, Stachon P, Zotzmann V, Bode C, Biever PM, Staudacher D, Wengenmayer T, Graf E, Duerschmied D (2021) Cytokine adsorption in patients with severe COVID-19 pneumonia requiring extracorporeal membrane oxygenation (CYCOV): a single centre, open-label, randomised, controlled trial. Lancet Respir Med. https://doi.org/10.1016/S2213-2600(21)00177-6

5. Bonavia A, Groff A, Karamchandani K, Singbartl K (2018) Clinical utility of extracorporeal cytokine hemoadsorption therapy: a literature review. Blood Purif 46:337-349 